\title{
Die richtungweisende Führungsrolle der Europäischen Union in der internationalen Klimapolitik
}

\section{Einleitung}

In den vergangenen Jahren hat die Europäische Union (EU) eine Führungsrolle in der internationalen Klimapolitik eingenommen (Michaelowa 2006; Oberthür, Roche Kelly 2008; Lindenthal 2009; Haug, Berkhout 2010; Roche Kelly, Oberthür, Pallemaerts 2010). Zum einen wäre das Kyoto-Protokoll verschiedenen Autoren zufolge ohne die EU und ihre aktive Rolle in den internationalen Klimaverhandlungen nicht zustande gekommen (Gupta, Ringius 2001: 294; Schreurs, Tiberghien 2007: 22). Zum anderen wird konstatiert, dass die EU im Gegensatz zu vielen anderen Industriestaaten frühzeitig mit Maßnahmen zur Reduktion von Treibhausgasemissionen begonnen hat (Fischer 2009: 108-109). Hier gilt als prominentes Beispiel der europäische Emissionshandel (z.B. Lindenthal 2009: 266).

Seit der Kopenhagener Klimakonferenz im Dezember 2009 scheint der von Sebastian Oberthür und Marc Pallemaerts diagnostizierte Gleichlauf des internationalen Klimaregimes und der EU-Klimapolitik jedoch nicht mehr gegeben zu sein (Oberthür, Pallemaerts 2010: 27, 52.f.). Zwar hatte die EU im Vorfeld der Verhandlungen konkrete Vorschläge für eigene Reduktionsverpflichtungen in einem post-Kyoto-Abkommen unterbreitet. Das schwache Ergebnis des Kopenhagener Klimagipfels, den rechtlich unverbindlichen und inhaltlich vagen Kopenhagener Akkord, konnte die EU allerdings nicht verhindern. Ausgehend von dieser Beobachtung stellen wir folgende Frage: Welche Möglichkeiten bestehen für die EU, auch nach den gescheiterten Verhandlungen in Kopenhagen eine Führungsrolle in der internationalen Klimapolitik auszuüben?

Nach einer kurzen Diskussion des Konzepts der Führungsrolle zeigen wir, wie die EU bisher ihre Führungsrolle in der internationalen Klimapolitik ausgeübt hat. Im Anschluss diskutie ren wir, in welcher Form die EU nach Kopenhagen erneut eine Führungsrolle übernehmen kann. Unser Hauptargument ist, dass die EU ihre Führungsrolle trotz der in Kopenhagen sichtbar gewordenen fundamentalen Machtverschiebungen durch richtungsweisende Initiativen zurück gewinnen könnte (vgl. Vogler, Stephan 2007). Hierfür müssen die Politiken in unterschiedlichen Feldern stärker auf den Klimaschutz ausgerichtet werden, um einen möglichst großen Vorbildcharakter der EU-Politiken zu erreichen. Inwieweit die EU ihre Politiken bereits auf den Klimaschutz ausgerichtet hat, analysieren wir anhand von drei Politikfeldern, die für den Klimawandel relevant sind, sich aber in ihrem Vergemeinschaftungsgrad unterscheiden: Der Energie-, Entwicklungs- und Agrarpolitik. 


\section{Das Konzept der Führungsrolle}

Seit Ende der 1980er Jahre wird das Konzept der Führungsrolle in der politikwissenschaftlichen Forschung zu internationalen Regimen intensiv diskutiert (vgl. Skodvin, Andresen 2006). Verschiedene Wissenschaftler sind der Frage nachgegangen, ob und inwieweit eine von Staaten oder Staatengruppen ausgeübte Führungsrolle ${ }^{1}$ zur Überwindung von Kooperationsproblemen beitragen kann (Young 1989; Young 1991; Underdal 1994). Dabei wird unter einer Führungsrolle zuerst einmal die Fähigkeit und Bereitschaft eines Akteurs verstanden, sich für die Lösung eines kollektiven Handlungsproblems einzusetzen.

Bisher gibt es jedoch weder eine allgemein akzeptierte Definition des Begriffs, noch besteht Konsens über die Klassifizierung verschiedener Formen und Ausprägungen der Führungsrolle (vgl. Grubb, Gupta 2000: 18). Zudem konstatieren einige Autoren, dass mit der Verwendung des Begriffs schwerwiegende analytische Probleme verbunden sind (z.B. Skodvin, Andresen 2006). Dabei gilt es als größte Herausforderung, die reine Verfolgung von Eigeninteressen durch den Leader analytisch von der Ausübung einer Führungsrolle zu trennen. So konnte bislang nicht zufriedenstellend dargelegt werden, wie Fälle zu bewerten sind, in denen die Interessen des Leaders mit dem gemeinsamen Ziel der Akteure deckungsgleich sind. (Young 1991: 293; Underdal 1994: 185-187; Malnes 1995: 93-95; Skodvin, Andresen 2006: 16-18).

Nach unserem Verständnis ist das Eigeninteresse des Leaders an der Kooperation eine notwendige Voraussetzung dafür, dass dieser eine Führungsrolle einnimmt. In Anlehnung an Malnes und Underdal fassen wir allerdings Handlungen, die ausschließlich der Verfolgung nationaler Interessen dienen, nicht unter den Begriff der Führungsrolle (vgl. Underdal 1994: 178; Malnes 1995: 94). Folglich ist für uns mit der Rolle des Leaders die Bereitstellung eines öffentlichen Guts bzw. ein gemeinsames Ziel der Akteure eng verbunden. Zweitens gehen wir davon aus, dass der Leader aufgrund seiner herausgehobenen Position im internationalen Regime Einfluss auf andere Akteure nehmen kann. Drittens kann eine Führungsrolle nicht ausschließlich auf Zwang oder der Anwendung von Gewalt basieren (Underdal 1994: 179), sondern muss sich auf die gemeinsamen Interessen der Akteure stützen. Viertens ist eine Führungsrolle durch eine gewisse Dauerhaftigkeit, also Stabilität, gekennzeichnet (Underdal 1994: 179).

Unter Rückgriff auf die Klassifizierung von Skodvin und Andresen unterscheiden wir drei Arten der Ausübung einer Führungsrolle: (i) die machtbasierte, (ii) die unternehmerische und (iii) die richtungsweisende Führungsrolle (Skodvin und Andresen 2006: 14-15). Diese drei Formen bedienen sich unterschiedlicher Instrumente und haben jeweils andere Voraussetzungen. Für die machtbasierte Form setzt der Leader auf „Zuckerbrot und Peitsche“ (Underdal 1994: 186; siehe auch Malnes 1995) und greift auf sein Machtpotential zurück, um das gemeinsame Ziel der Verhandlungsakteure durchzusetzen. Diese Form der Führungsrolle erfordert eine starke Ressourcenbasis und das Potential, die eigene Macht instrumentell einzusetzen ${ }^{2}$.

1 Hier gleichgesetzt mit Leadership.

2 Wir beschränken den Machtbegriff hier auf seine instrumentelle Ausprägung, um eine bessere Abgrenzung zur unternehmerischen und richtungsweisenden Führungsrolle zu gewährleisten. Ein erweiterter Machtbegriff würde alle drei Spielarten als Ausprägung von Macht verstehen. 
Bei der unternehmerischen Form baut der Leader auf sein politisches Verhandlungsgeschick, um sowohl die politische Agenda als auch die Struktur von Verhandlungen und Entscheidungsprozeduren zu beeinflussen. Hier sorgt sich der Leader um einen Interessensausgleich zwischen allen wichtigen Verhand lungspartnern, um einen möglichst breit akzeptierten Verhand lungsausgang zu erreichen (Young 1991; Underdal 1994; Grubb, Gupta 2000).

In der richtungsweisenden Form schließlich versucht der Leader, durch ,unilaterale Handlungen“ (Underdal 1994) anderen Akteuren die Überlegenheit und den Vorbildcharakter bestimmter Lösungsansätze zu demonstrieren. Diese Form der Führungsrolle basiert auf überzeugenden und glaubwürdigen Politiken und Initiativen des Leaders (Grubb, Gupta 2000: 21), an dem sich andere Akteure orientieren und ihr Verhalten ausrichten. Im Folgenden beschreiben wir anhand dieser Klassifizierung die Entwicklung der EU zum Leader in der internationalen Klimapolitik von den 1990er Jahren bis zu den Verhandlungen in Kopenhagen.

\section{Die Führungsrolle der EU bis Kopenhagen}

Bereits in den frühen 1990er Jahren hat die EU eine Führungsrolle in den internationalen Klimaverhandlungen eingenommen (Roche Kelly, Oberthür, Pallemaerts 2010: 13f.). So forderte sie im Vorfeld der Konferenz der Vereinten Nationen über Umwelt und Entwicklung 1992 in Rio de Janeiro verbindliche Verpflichtungen zur Stabilisierung der Treibhausgasemissionen von Industrieländern (Michaelowa 2006: 169; Lindenthal 2009: 140-143; Oberthür, Pallemaerts 2010: 30). Sie trug ebenfalls wesentlich zur Ausgestaltung der Klimarahmenkonvention bei, die auf dieser Konferenz von über 150 Staaten unterzeich net wurde (Lindenthal 2009: 159-161). Zudem hatte die EU entscheidenden Anteil daran, dass 1997 das Kyoto-Protokoll beschlossen wurde (Roche Kelly, Oberthür, Pallemaerts 2010: 13). Dieses Zusatzprotokoll zur Klimarahmenkonvention gibt verbindliche Ziele für die Reduktion der Treibhausgasemissionen von Industrieländern vor. Zwar gelang es der EU nicht, die USA zur Ratifizierung des Protokolls zu bewegen. Aber sie konnte mehrere andere Staaten dazu bringen, verbindliche Reduktionsverpflichtungen einzugehen, unter anderem Japan und Russland (Skodvin, Andresen 2006: 20-22; Schreurs, Tiberghien 2007: 23). Die EU hat demnach die Klimaverhandlungen bis Ende der 1990er Jahre durch Verhandlungsgeschick und den Ausgleich verschiedener Interessen vorangebracht, was wir als unternehmerische Form der Führungsrolle bezeichnen.

Seit Ende der 1990er Jahre hat die EU zudem verschiedene interne Klimaschutzmaßnahmen eingeleitet (vgl. Oberthür, Pallemaerts 2010: 43-52) und mehrere Initiativen mit Vorbildcharakter auf den Weg gebracht, die Lösungsansätze für die Eindämmung und Bewältigung des Klimawandels aufzeigen. Ein häufig genanntes Beispiel hierfür ist der 2003 vom Europäischen Parlament und dem EU-Ministerrat beschlossene europäische Emissionshandel (z.B. Lindenthal 2009: 266), der mehreren Autoren zufolge die Glaubwürdigkeit der EU-Klimapolitik erhöhte (Schreurs, Tiberghien 2007: 20; Lindenthal 2009: 266; Oberthür, Pallemaerts 2010: 28; Roche Kelly, Oberthür, Palle- 
maerts 2010: 13). Diese vorbildhafte Einführung von Maßnahmen durch die EU klassifizieren wir als richtungsweisende Form der Führungsrolle.

\section{Die Führungsrolle der EU nach Kopenhagen}

Es wäre zu kurz gegriffen, allein die schwedische EU-Ratspräsidentschaft oder die dänische Verhandlungsführung für das weithin konstatierte Scheitern des Kopenhagener Klimagipfels verantwortlich zu machen (vgl. Haug, Berkhout 2010). Vielmehr haben die Verhandlungen grundlegende Machtverschiebungen im internationalen Klimaregime sichtbar werden lassen. Aufgrund dieser fundamentalen Veränderungen können die Interessensgegensätze der einzelnen Akteure im internationalen Klimaregime nicht länger durch einseitige Angebote der EU überbrückt werden (vgl. Lederer 2010). Die bisher von der EU ausgeübte unternehmerische Form der Führungsrolle ist also seit Kopenhagen aufgrund struktureller Machtverschiebungen in der internationalen Klimapolitik schwerer oder gar nicht mehr umsetzbar, wodurch im Gegenzug die richtungsweisende Form der Führungsrolle besondere Relevanz erhält. Gelingt es der EU, überzeugende Klimaschutzmaßnahmen zu entwickeln und anderen Staaten Lösungsansätze für eine effiziente Reduktion von Treibhausgasemissionen zu demonstrieren, kann sie die internationale Klimapolitik weiter voranbringen. Zwar garantieren vorbildhafte Politiken nicht, dass andere Staaten diese auch übernehmen. Sie sind jedoch derzeit die einzig erfolgversprechende Möglichkeit, andere Staaten von der Einführung weiterer Maßnahmen gegen den Klimawandel zu überzeugen. Im Folgenden analysieren wir, inwiefern es der EU in der Energie-, Entwicklungs- und Agrarpolitik bereits gelungen ist, Maßnahmen mit Vorbildcharakter zu entwickeln.

\section{EU-Energiepolitik}

Im März 2007 verabschiedeten die Staats- und Regierungschefs der EU einen Aktionsplan, in dem die langfristigen energie politischen Ziele der EU formuliert sind (Europäischer Rat 2007). Ein knappes Jahr später veröffentlichte die Europäische Kommission das Klima- und Energiepaket (Europäische Kommission 2008). Mit diesem Maßnahmenpaket strebt die EU an, die enge Beziehung zwischen der Klima- und Energiepolitik hervorzuheben (vgl. Howes 2010: 125). Auf Grundlage dieses Pakets erließen das europäische Parlament und der Rat der EU in den folgenden Monaten mehrere Richtlinien, die laut verschiedener Autoren die Glaubwürdigkeit der EU-Klimapolitik erhöht haben (Schreurs, Tiberghien 2007: 20; Lindenthal 2009: 266; Oberthür, Pallemaerts 2010: 28; Roche Kelly, Oberthür, Pallemaerts 2010: 13). Nachfolgend wird untersucht, inwieweit die zentralen Elemente dieses Pakets Vorbildcharakter für andere Staaten besitzen.

Kurz nach der Veröffentlichung des Klima- und Energiepakets erließen das Europäische Parlament und der Rat der EU im April 2009 die sogenannte Erneuerbare-Energien-Richtlinie (Europäisches Parlament und Rat der EU 2009b). Diese Richtlinie schreibt den EU-Mitgliedstaaten individuelle Richtziele zur Steigerung des 
Anteils von erneuerbaren Energien an ihrem Energieverbrauch im Strom-, Heiz- und Verkehrssektor vor. Auf diese Weise versucht die EU ihr Ziel zu erreichen, den Anteil von erneuerbaren Energien am Gesamtenergieverbrauch der EU bis 2020 auf 20 Prozent zu erhöhen. Wenige Wochen später legte die Europäische Kommission einen Entwurf vor, auf dessen Basis die Mitgliedstaaten ihre jeweiligen Nationalen Aktionspläne zur Förderung von erneuerbaren Energien für die kommenden Jahre bis Mitte 2010 formulieren sollen (Europäische Kommission 2009b). Im Anschluss daran müssen die EU-Mitgliedstaaten über die Umsetzung ihrer Pläne alle zwei Jahre Bericht erstatten.

Diese jüngsten Initiativen der EU zur Förderung von erneuerbaren Energien werden von verschiedenen Autoren als erfolgversprechende Maßnahmen zur Reduktion von Treibhausgasemissionen bewertet (z.B. Roche Kelly, Oberthür, Pallemaerts 2010: 18). Hervorgehoben wird dabei vor allem der Fortschritt, den die EU bei der Förderung erneuerbarer Energien in den vergangenen Jahren gemacht hat (Howes 2010: 124-145). Allerdings hat die Erneuerbare-Energien-Richtlinie im Verhandlungsprozess einige Abschwächungen erfahren. Ursprünglich sah die Richtlinie beispielsweise vor, dass alle EU-Mitgliedstaaten, die ihre individuellen Zwischenziele verfehlen, automatisch Geldstrafen auferlegt bekommen (EurActiv 2010c). Dieser verbindliche Sanktionsmechanismus wurde jedoch nicht in die Richtlinie aufgenommen. Wenn es der EU trotzdem gelingt, 20 Prozent ihres Energiebedarfs 2020 mit er neuerbaren Energien zu decken, was aktuelle Schätzungen der Europäischen Kommission voraussagen (Europäische Kommission 2010), kann sie anderen Staaten ein überzeugendes Beispiel liefern für ein effektives Instrument zur Reduktion von Treibhausgasemissionen.

Schon vor der Ankündigung der EU, 20 Prozent ihres eigenen Energieverbrauchs gegenüber den Prognosen für 2020 einsparen zu wollen (Europäischer Rat 2007), hatten das Europäische Parlament und der Rat der EU zwei Maßnahmen zur Steigerung der Energieeffizienz auf den Weg gebracht (vgl. Oberthür, Pallemaerts 2010: 43). Zum einen hatten das Europäische Parlament und der Rat der EU 2005 die sogenannte Ökodesign-Richtlinie erlassen, mit der die Energieeffizienz von Haushaltsgeräten erhöht werden soll (Europäisches Parlament und Rat der EU 2005). Zum anderen verabschiedeten diese beiden EU-Institutionen 2006 eine Richtlinie zur Festlegung nationaler Energieeinsparungsrichtwerte (Europäisches Parla ment und Rat der EU 2006). In den darauf folgenden Jahren hat die EU einige spezielle Verordnungen erlassen, um die Ökodesign-Richtlinie mit konkreten Vorschriften zu erweitern. Ein Beispiel hierfür ist die Verordnung der Europäischen Kommission zur schrittweisen Abschaffung von Glühbirnen (Europäische Kommission 2009c).

Industrieverbände bemängeln an diesen Vorschriften aller dings, dass die EU bisher keine Regelungen getroffen habe, um die Einhaltung der Effizienzstandards zu kontrollieren und Unternehmen zu sanktionieren, die falsch deklarierte Produkte verkaufen (vgl. EurActiv 2010a; Schröder 2010). Umweltgruppen kritisieren zudem die mangelnde Zielumsetzung der EU, die Energieeffizienz um 20 Prozent zu steigern (Germanwatch 2008; Klima-Allianz 2010). Ihr zentraler Kritikpunkt ist, dass weder die Richtlinie zur Festlegung nationaler Einsparungsrichtwerte, noch das 20-Prozent-Ziel bislang für die EU-Mitgliedstaaten rechtlich verbindlich seien, sondern lediglich Richtwerte dar stellten. Darüber hinaus sprach sich der EU-Ministerrat Ende Mai 2010 gegen strengere Vorschriften zur Energieeinsparung aus, um die Wettbewerbsfähigkeit der europäischen 
Industrie nicht zu gefährden (vgl. EurActiv 2010b; Rat der EU 2010). Dies zeigt, dass die Klimaschutzziele der EU zumindest teilweise hinter ihre Wirtschaftsinteressen zurückgetreten sind. Eine konsequente Umsetzung der EU-Initiativen zur Steigerung der Energieeffizienz steht somit noch aus.

Der Emissionshandel der EU wird von einigen Autoren als das „Prunkstück“ der EU-Klimapolitik angesehen (Oberthür, Pallemaerts 2010: 42). Die Grundidee des europäischen Emissionshandels ist es, die für die EU-Mitgliedstaaten im Kyoto-Protokoll festgeschriebenen Verpflichtungen zur Reduktion von Treibhausgasemissionen möglichst kostengünstig zu erreichen. Europäische Unternehmen können im Rahmen des europäischen Emissionshandels Emissionsrechte frei handeln. Dadurch soll ein Anreiz für Unternehmen geschaffen werden, Treibhausgasemissionen einzusparen (Europäisches Parlament, Rat der EU 2003). Im April 2009 hat die EU eine Reform ihres Emissions handels eingeleitet (Europäisches Parlament, Rat der EU 2009c). Mit dieser Maßnahme strebt sie an, mehrere Schwachstellen der ersten beiden Handelsperioden von 2005 bis 2007 und von 2008 bis 2012 zu beseitigen (vgl. Birger Skjaerseth, Wettestad 2010: 80). Die wichtigste vorgesehene Neuerung für die dritte Handelsperiode von 2013 bis 2020 ist eine EU-weite Gesamtobergrenze für Treibhausgasemissionen statt nationaler Allokations programme. Zudem soll diese Obergrenze schrittweise reduziert werden statt sie, wie bisher, über die gesamte Handelsperiode konstant zu halten. Darüber hinaus soll das Auktionsprinzip gegenüber der kostenlosen Verteilung von Emissionsrechten gestärkt werden (Europäisches Parlament, Rat der EU 2009c).

Durch diese Reformen wird in der dritten Handelsperiode ein größerer Anteil der Treibhausgasemissionen vom europäischen Emissionshandel abgedeckt. Zudem vergrößern sich für Unternehmen die Anreize, tatsächlich Treibhausgasemissionen einzusparen. Vielen Autoren gehen die Reformen jedoch nicht weit genug. Sie kritisieren, dass auch in der dritten Handelsperiode ein großer Teil der Emissionsrechte an die Unternehmen frei verteilt werde (Birger Skjaerseth, Wettestad 2010: 86; Oberthür, Pallemaerts 2010: 47-48). Zum einen erhalten stromerzeugende Unternehmen aus mehreren neuen EU-Mitgliedstaaten einen Großteil der Emissionsrechte kostenlos. Zum anderen sind exportorientierte Unternehmen, die der Gefahr von Carbon Leakage ${ }^{3}$ unterliegen, vom Auktionsprinzip weitgehend ausgenommen. Ebenfalls wurden dem produzierenden Gewerbe Zugeständnisse eingeräumt. Hier kommt es voraussichtlich erst $2027 \mathrm{zu}$ einer vollständigen Versteigerung der Emissionsrechte (Fischer 2009: 112; Oberthür, Pallemaerts 2010: 48).

Diese Ausnahmeregelungen sind auf den Druck einzelner EU-Mitgliedstaaten zurückzuführen. Während sich beispiels weise Polen dagegen wehrte, seine Kohlekraftwerke unter das Auktionsprinzip zu stellen, setzte sich Deutschland für die freie Zuteilung von Emissionsrechten an exportorientierte Industrien ein (Birger Skjaerseth, Wettestad 2010: 80). Dies schwächt die Effektivität des europäischen Emissionshandels. Sollte es der EU gelingen, die Sonderbehandlung bestimmter Industriezweige aufzuheben, kann der europäische Emissionshandel anderen Staaten und Regionen als

3 Mit diesem Begriff wird die Verlagerung von Produktion und Emissionen ins Ausland bezeichnet. 
Vorbild und Anknüpfungspunkt die nen (vgl. Wissenschaftlicher Beirat der Bundesregierung Globale Umweltveränderungen 2010).

\section{EU-Entwicklungspolitik}

Die Auseinandersetzungen um gerechte Finanzierung und Ausgleichszahlungen zwischen Industrie-, Schwellen-, und Entwicklungsländern konstituieren eine der primären Konfliktlinien in den internationalen Klimaverhandlungen. Die Entwicklungsländer haben deutlich gemacht, dass ohne die Bereitstellung zusätzlicher Mittel über die bestehenden Entwicklungshilfe-Zusagen der Millenniumsziele von 0,7 Prozent des Bruttoinlandseinkommens hinaus und ohne einen verstärkten Technologietransfer das angestrebte post-Kyoto-Abkommen nicht zustande kommt. Daher ist die Ausübung einer richtung sweisenden Führungsrolle der EU in der Entwicklungspolitik besonders wichtig, um Lösungen aufzuzeigen und voran zu treiben. Um vorbildhaft Klimaschutz in die EU- Entwicklungszusammenarbeit zu integrieren, müssen sowohl Anpassungsals auch Reduktionsmaßnahmen abgedeckt werden. Zum einen müssen die betroffenen Länder bei der Bewältigung und Begrenzung von Desertifikation, Wassermangel und Naturkatastrophen unterstützt werden. Zum anderen muss der Ausstoß von Treibhausgasen in Entwicklungsländern begrenzt und vermindert werden, etwa durch Technologietransfer für eine carbon-arme wirtschaftliche Entwicklung (Peskett et al. 2009).

Seit 1992 haben die meisten Akteure der internationalen Entwicklungszusammenarbeit mit dem sogenannten Klima-Mainstreaming begonnen (Gupta, Persson, Olsson 2010). Auf der Ebene der Empfängerländer wurden für eine verbesserte Abstimmung und für das Mainstreaming der nationalen Entwicklungspolitiken mit Querschnittthemen wie Armutsbekämpfung und Geschlechter-Gerechtigkeit in den vergangenen Jahren unterschiedliche Instrumente entwickelt. Das Mainstreaming mit diesen Querschnittthemen dient zum Teil als Konditionalität für die Mittelvergabe. Es ist damit fest verankert in nationalen Entwicklungsplänen, die im Rahmen verschiedener Entwicklungsprogramme von den Empfängerländern erstellt und von den Geberinstitutionen abgenommen werden wie z.B. bei den Armutsbekämpfungsstrategien der Weltbank oder bei den Nationalen Strategien für Nachhaltige Entwicklung. Hier setzt auch der diesbezügliche EU-Aktionsplan an, mit dem vor allem die Integration nationaler Klima-bezogenen Maßnahmen in diese umfassenden nationalen Strategie-Instrumente vorangetrieben werden soll (Peskett et al. 2009).

Die EU hat seit 1998 verschiedene Initiativen gestartet, um Klimaschutz und Entwicklungszusammenarbeit zu verbinden und zu finanzieren. Beispiele hierfür sind der EU Aktionsplan Klimaänderungen und Entwicklungszusammenarbeit von 2004, die Globale Allianz gegen den Klimawandel und das oben be reits diskutierte Klima- und Energiepaket der EU. Weitere Instrumente, die auf der EU-Geberseite Klima-Mainstreaming ermöglichen sollen, sind die Umweltverträglichkeitsprüfung und die Strategische Umweltprüfung (Peskett et al. 2009). Die tatsächliche Höhe der bisherigen EU-Mittel für Klimaschutz- und Anpassungsmaßnahmen ist schwer zu bestimmen, da es keine klare Definition davon gibt, was Klima-relevante Finanzierung ist (Ayers, Huq, Chandani 2010). Ebenfalls ist unklar, ob die Mittel der Gemeinschafts- 
ebene von denen der Mitglied staaten getrennt betrachtet werden können. Ebenso ungeklärt ist der tatsächliche Mittelbedarf. Dieser variiert zwischen vier und über 100 Milliarden US-Dollar pro Jahr (Ayers, Huq, Chandani 2010).

Für die Verringerung des $\mathrm{CO}_{2}$-Ausstoßes sagte das Sekretariat der Klimarahmenkonvention 176 Milliarden US-Dollar an zusätzlichem Investitionsbedarf bis 2030 voraus. Im Hinblick darauf fordern Mitglieder des Europäischen Parlaments zwei Milliarden Euro bis 2010 und zwischen fünf und zehn Milliarden Euro bis 2020 zusätzlich (Peskett et al. 2009). Bei allen Berechnungen und Forderungen scheint der Finanzierungs bedarf im Vergleich zu den bisherigen EU-Mitteln enorm zu sein. Gleichzeitig stagnieren die Entwicklungshilfe-Ausgaben der Mitgliedstaaten in den vergangenen Jahren oder sind teil weise sogar rückläufig. Nur die wenigsten EU-Mitgliedstaaten stellen zusätzliche Mittel zu denen im Rahmen der Milleniumsziele geforderten 0,7 Prozent des Bruttonationalein kommens für Klimaschutz-Maßnahmen bereit (Ayers, Huq, Chandani 2010).

Die größten Erwartungen für die Finanzierung liegen daher auf der Generierung zusätzlicher Mittel aus dem europäischen Emissionshandel bzw. dem Mechanismus für umweltverträgliche Entwicklung und der Einführung eines globalen Finanzierungsmechanismus. Laut einem Bericht des Sekretariats der Klimarahmenkonvention müssen 85 Prozent der benötigten Finanzmittel aus privaten Quellen bezogen werden (United Nations Frame work Convention on Climate Change 2007). Dementsprechend richten sich der Globale Fond für Effizienz und Erneuerbare Ener gien, die Unterstützungsmaßnahmen der Europäischen Investitionsbank und die Strukturen des europäischen Emissionshandels vor allem auf die Mobilisierung und Akquirierung privatwirtschaftlicher Mittel aus (Peskett et al. 2009).

Aber auch im Bereich der Finanzierung hat die EU noch keine entscheidende Abstimmungs- und Koordinierungsbemühungen mit anderen internationalen Finanzierungsinstrumenten erbracht, von denen seit 2007 mindestens 14 neue aufgelegt wurden. Besonders intensiv werden in jüngster Zeit neue vertikale Fonds diskutiert, die durch die Globale Umweltfazilität auf der Ebene der Vereinten Nationen gemeinsam mit der Weltbank administriert werden sollen. Aus den Entwicklungsländern werden angesichts der institutionellen Verankerung der Fonds Bedenken in Bezug auf die Repräsentation der Empfängerländer, die komplizierten Antragsprozeduren und die Rückzahlung der Zuwendungen laut (Peskett et al. 2009; Ayers, Huq, Chandani 2010). In dieser Debatte hat sich die EU bisher nicht entscheidend zu Wort gemeldet, obwohl es Einzahlungen in unterschiedliche Fonds der Klimarahmenkonvention gab und mindestens fünf Millionen Euro in die Forest Carbon Partnership Facility der Weltbank geflossen sind.

Viel eher scheint die EU einen Mittelweg zwischen der Komplexität bestehender Klimafazilitäten und eigener Finanzierung und Steuerung zu suchen. Dabei ist vor allem die bereits erwähnte Globale Allianz gegen den Klimawandel interessant, die die Koordinierung der unterschiedlichen Klimaschutzmaßnahmen in der europäischen Entwicklungszusammenarbeit übernehmen soll (Peskett et al. 2009). Im Rahmen dieser Allianz sollen vor allem auch Allgemeine Haushaltszuschüsse als Instrument für die Unterstützung der Empfängerländer im Bereich des Klimaschutzes vorangetrieben werden. Diese Form der Finanzierung soll der Vertrauensbildung in den Enzwicklungsländern dienen und eine flexible Verwendung der Mittel in Abstimmung mit anderen 
Entwicklungsmaßnahmen ermöglichen. Damit positioniert sich die Allianz in einer kontroversen Debatte über adäquate Instrumente in der Entwicklungszusammenarbeit zwischen den EU-Mitgliedstaaten und nimmt hier in Bezug auf viele andere Klimafonds mit seiner Befürwortung von Budgethilfe eine Sonderrolle ein. Bisher sind noch nicht alle Mitgliedstaaten bereit, diesen eigenen Weg der EU mitzuge hen und verlangen eine Darlegung des Mehrwerts eines eigenen Fonds auf EU-Ebene, bevor sie Mittel einzahlen (Peskett et al. 2009). Vor allem Großbritannien möchte bis auf weiteres seine bilateralen Instrumente wie den Environmental Transformation Fund nutzen und keine Gelder für die Allianz bereitstellen (Uni ted Kingdom Parliament 2008).

Bei allen Maßnahmen in diesem Bereich stellt sich abgesehen von der Finanzierung die Frage der Vereinbarkeit mit anderen, teilweise gegenläufigen Zielen und Grundsätzen der Entwicklungszusammenarbeit (Gupta, Persson, Olsson 2010). Koordinierung, Kohärenz und Komplementarität sind durch die Erklärung von Paris 2005 als Arbeitsgrundsätze für die Geberkoordinierung und die Erhöhung der Eigenverantwortlich keit in den Empfängerländern in der internationalen Entwicklungszusammenarbeit festgelegt worden (Bundesministerium für wirtschaftliche Zusammenarbeit und Entwicklung 2010). So wird beispielsweise die vom Europäischen Rechnungshof 2006 kritisierte, unzureichende Integration der sogenannten Umweltprofile in die übergeordneten Länderstrategie-Papiere von der Europäische Kommission auf die fehlende Priorität des Klimaschutzes gegenüber anderen Entwicklungszielen und Politiken in den Entwicklungsländern zurückgeführt. Auch eine aktuelle Studie bestätigt das Primat der allgemeiner Entwicklungsziele vor Anpassungsmaßnahmen an den Klimawandel, Reduktion von Treibhausgasen steht in der Zielhierarchie der Entwicklungsländer an letzter Stelle (Shrivastava, Goel 2010). Ein hierarchisches Vorantreiben des Klimaschutzes in den Empfängerländern stellt dabei keine Lösung dar, da dies mit Bemühungen zur Stärkung der entwicklungspolitischen Eigenverantwortung der Länder sowie mit dem Ziel, auf politische Konditionalitäten zu verzichten, nicht übereinstimmt (Peskett et al. 2009).

Die Kohärenz von Klimapolitiken und Entwicklungszusammenarbeit wird besonders kontrovers diskutiert in den Be reichen Bio-Diesel und Emissionshandel. Vor allem wurden Bedenken laut, dass die Erreichung des 10-Prozent-Reduktionsziel in Bezug auf den europäischen Spritverbrauch vor allem durch die Umstellung auf Bio-Diesel erreicht werden soll, was zu Ab holzungen von Regenwald in Entwicklungsländern und zur Umwidmung von landwirtschaftlicher Nahrungsmittelproduk tion und damit zu Nahrungsmittelknappheit führen kann. Hier wurde teilweise Abhilfe geschaffen durch Standard-Setzung für die Gewinnung von Bio-Diesel (Peskett et al. 2009).

Ähnliche Bedenken wurden auch in Bezug auf die Integration von Aufforstungsbemühungen in die $\mathrm{CO}_{2}$-Handels-Fazilitäten laut. Die dadurch gesetzten finanziellen Anreize könnten dazu führen, dass Regierungen forstwirtschaftliche und landwirtschaftliche Nutzungen zugunsten von Wiederaufforstungsprogrammen untersagen, was sich negativ auf die Nahrungsmittelproduktion auswirken kann. Aus diesem Grund hat die EU bisher Wald- und Forstwirtschaft nicht in den europäischen Emissionshandel integriert. Trotzdem hat die EU damit begonnen, internationale Aufforstungs- und Waldschutzbemühungen zu unterstützen und zu beeinflussen (Peskett et al. 2009). Hier entstehen vor 
allem Synergien zwischen den Politiken des EU-Programms Forest Law Enforcement, Governance and Trade und dem UN-Programm Reducing Emissions from Deforestation and Degradation.

\section{EU-Agrarpolitik}

Im Klima- und Energiepaket der EU spielt die Gemeinsame Agrarpolitik bisher kaum eine Rolle, obwohl dies der Politik bereich ist, der nach allgemeiner Einschätzung den höchsten Grad an Integration aufweist und in dem die EU-Institutionen die größten Finanzströme lenken. (Einen Überblick hierzu geben Núnez, Egenhofer, Behrens 2009).

Die bisherigen Maßnahmen zielten eher auf lokalen Umweltschutz und die Erhaltung bzw. Förderung von Biodiversität ab als auf den Schutz des internationalen Klimas. Da es bislang nur wenige Studien zu diesem Bereich gibt, stützen sich die folgenden Ausführungen primär auf Dokumente der Europäischen Kommission.

Wenn der Klimawandel im Zusammenhang mit der Ge meinsamen Agrarpolitik Erwähnung findet, werden in erster Linie die Herausforderungen für Anpassungsmaßnahmen - besonders in Südeuropa - betont, da prognostiziert wird, dass es in Europa zu verstärkter Wasserknappheit, Rückgang der Boden qualität und einer Ausweitung von Parasiten und Krankheiten kommen wird. (Eine gute Zusammenfassung der Auswirkungen des Klimawandels findet sich bei Olesen, Bindi 2002).

Auch das Thema Nahrungsmittelsicherheit wird immer wie der angesprochen und die Notwendigkeit betont, neues Saatgut und neue Techniken zu entwickeln (Europäische Kommission 2009a). Zur Reduktion von Treibhausgasemissionen hat die EU bisher jedoch kaum Maßnahmen getroffen, obwohl der Anteil der Emissionen aus der Landwirtschaft mit circa zehn Prozent vergleichsweise hoch ist und 2007 bei insgesamt 462 Millionen Tonnen $\mathrm{CO}_{2}$ lag (Europäische Kommission 2009a: 7). Die absoluten Emissionen aus der Landwirtschaft sinken zwar seit einigen Jahren, wenngleich der Ertrag an landwirtschaftlichen Gütern gestiegen ist. Dieser Trend wird aber ohne eine Umstel lung der praktizierten Methoden nicht fortgesetzt werden kön nen (Europäische Kommission 2009a: 7).

Da die Hauptemittenten in der Landwirtschaft - die vielen kleinen und unterschiedlichen Produzenten - technisch schwer zu erfassen sind, werden sie nicht in den europäischen Emissionshandel integriert ${ }^{4}$. Stattdessen hat die EU pauschal vorgegeben, die Treibhausgasemissionen aus der Landwirtschaft um zehn Prozent zu senken (Europäisches Parlament, Rat der EU 2009a). Die diesbezügliche Entscheidung des europäischen Parlaments und des Rats der EU lässt jedoch einen hohen Grad an Flexibilität zu und es bleibt den Mitgliedstaaten überlassen, wie sie die erforderlichen Reduktionen in der Landwirtschaft erzielen. Technisch ist dies vor allem in drei Bereichen möglich:

(i) durch die Optimierung bei der Verwendung von Düngemitteln;

\footnotetext{
4 Neuseeland ist bislang das einzige Land, das plant die Landwirtschaft in ein Emission shandelssystem einzubeziehen. Die neuseeländische Agrarwirtschaft wird somit ab 2012 auch zu Reduzierungen ihrer Treibhausgasemissionen im Rahmen des nationalen Cap gezwungen sein.
} 
(ii) durch Reformen bei der Tierhaltung und

(iii) durch einen ökologischen Umgang mit Tierexkrementen.

In einem weiteren Kontext muss die Absorptionsfähigkeit von Böden und Wäldern als $\mathrm{CO}_{2}$-Senken mit betrachtet werden, denn theoretisch können allein durch eine optimierte Nutzung von Agrarflächen bis zu 70 Millionen Tonnen $\mathrm{CO}_{2}$ eingespart werden (Europäische Kommission 2009a: 13).

Wenn die EU ihre Führungsrolle in der internationalen Klimapolitik fortsetzen will, muss sie ihre Agrarpolitik konse quenter nach ihren Klimaschutzzielen ausrichten. Einige wichtige Ansätze wurden bereits durchgeführt. Bereits zu Beginn der 1990er Jahre wurden viele Subventionen von direkten Produktionsbeihilfen auf Zahlungen zum Schutz und Erhalt von Flächen umgestellt. Aus Umwelt- und Klimaperspektive eindeutig problematische Subventionen - wie etwa Zuzahlungen für jedes gezüchtete Tier - wurden eingestellt und seit 2003 werden die Zahlungen direkt an die Landwirte geleistet. Durch regulative Politik wird zudem die Einbringung von Nitraten reduziert.

Für die gegenwärtige Planungsphase von 2007 bis 2013 räumt selbst die Europäische Kommission ein, dass die beste henden Maßnahmen nicht ausreichend sind. Sie versucht daher, in verschiedenen Bereichen nachzusteuern. Ein Beispiel hierfür ist die Arbeit an einer Richtlinie zum Schutz der Böden (Europäische Kommission 2006), die für eine verbesserte Ausnutzung des Absorptionspotential sorgen soll. Trotz dieser Maßnahmen gilt jedoch nach wie vor der Befund von Axel Michaelowa, dass eine wirklich progressive Klimapolitik im Agrarbereich nicht stattfindet, da landwirtschaftliche Lobbygruppen diese Policy-arena dominieren (Michaelowa 1998).

Die EU betont, dass mittelfristig eine substantielle Reduktion von Treibhausgasemissionen im Bereich der Gemeinsamen Agrarpolitik nur dann erreicht werden kann, wenn der Umbau zu einer nachhaltigen Landwirtschaft gelingt, die ökonomische, ökologische und soziale Aspekte vereint (Europäische Kommis sion 2009a: 25; vgl. Núnez, Egenhofer, Behrens 2009). Mögliche konkrete Schritte hierzu wären:

(i) der Schutz und Erhalt von landwirtschaftlichen Böden und Ausbau des Senkenpotentials - dem widersprechen jedoch Bemühungen der EU die beste henden LULUCF (Land-Use, Land-Use-Change and Forestry) Regeln des Kyoto Protokolls aufzuweichen;

(ii) ein besserer Austausch über Best Practices der Mitgliedstaaten;

(iii) verstärkte Forschung und Konsumenteninformationen sowie;

(iv) eine konkrete Einbeziehung von Klimaschutzmaßnahmen in ländliche Entwicklungspläne.

Außerdem sollte der Zusammenhang von Biodiversität und Klimaschutz mehr Beachtung finden und mittelfristig auch die europäische Landwirtschaft in das Emissionshandelssystem einbezogen werden. Die Vergabe von Subventionen muss zudem weiter reformiert werden, um Anreize zur intensiven Bewirtschaftung der Böden abzubauen (Boulanger, Messerlin 2009). Zwar hat die EU einen solch integralen Ansatz bereits mehrfach in den internationalen Verhandlungen gefordert, wenn es um den Schutz der tropischen Regenwälder geht. Für die gemeinsame Landwirtschaftspolitik im EU-Raum sind die diesbezüglichen Anstrengungen jedoch marginal und somit wenig richtungsweisend.

Insgesamt kann festgehalten werden, dass die EU-Initiativen in der EU-Agrarpolitik bislang nur sehr schwach auf den Klimaschutz ausgerichtet sind. Selbst die europäi- 
schen Institutionen sehen Bedarf für mehr Koordination und aktivere Gestaltung. Der Vorbildcharakter der EU-Initiativen für andere Staaten ist in diesem Politikfeld sehr gering.

\section{Fazit}

Das Scheitern der Kopenhagener Klimaverhandlungen hat gezeigt, dass die unternehmerische Führungsrolle der EU in der internationalen Klimapolitik aufgrund fundamentaler Machtverschiebungen im internationalen System ins Stocken geraten ist. Daher gewinnt die richtungsweisende Form der Führungsrolle besondere Relevanz für die EU. Wenn es der EU gelingt, vorbildhafte und überzeugende Maßnahmen zur Reduktion von Treibhausgasemissionen zu entwickeln, kann sie die internationale Klimapolitik weiter vorantreiben. Eine solche Politik garantiert zwar nicht, dass andere Staaten ähnliche Maßnahmen gegen den Klimawandel einführen. Sie ist jedoch derzeit die einzig erfolgversprechende Möglichkeit für die EU, eine Führungsrolle in der internationalen Klimapolitik auszuüben.

Unsere Analyse hat verdeutlicht, dass es der EU in den für den Klimawandel relevanten Politikfeldern bislang nur in eingeschränktem Maß gelungen ist, richtungsweisende Maßnahmen zu entwickeln. Das überraschende Ergebnis unserer Untersuchung ist dabei, dass die Maßnahmen in der EU-Agrarpolitik bisher am wenigsten auf den Klimaschutz ausgerichtet sind, obwohl dieses Politikfeld das am stärksten vergemeinschaftete ist. In den schwächer vergemeinschafteten Politikfeldern, der Entwicklungsund der Energiepolitik, sind die Maßnahmen der EU dagegen stärker auf den Klimaschutz ausgerichtet und besitzen Potential, um als Vorbild für andere Staaten zu dienen. Die EU hat allerdings in allen drei untersuchten Politikfeldern weiteren Spielraum, um ihre verschiedenen Politiken stärker auf den Klimaschutz auszurichten.

In der Energiepolitik hat die EU bereits einige Initiativen gestartet, die Vorbildcharakter für andere Staaten besitzen. Allerdings mangelt es vielen Maßnahmen der EU in der Energiepolitik noch an konkreten rechtsverbindlichen Vorschriften. Zudem treten die Klimaschutzziele der EU in der Energiepolitik teilweise hinter Wirtschaftsinteressen zurück, wie sich beispielsweise an den Ausnahmeregelungen im europäischen Emissionshandel ablesen lässt. In der EU-Entwicklungspolitik existieren ebenfalls einige Maßnahmen, die anderen Industriestaaten als Vorbild dienen können. Als problematisch werden allerdings die hohen administrativen Hürden für die Empfängerländer bei der Beantragung von Hilfsleitungen beurteilt. Weiterhin widersprechen sich oftmals Klimaschutz und konventionelle Entwicklungsziele, wobei das Vorantreiben von Entwicklung für viele Empfängerländer an erster Stelle steht. Bisher bleibt unklar, wie die EU-Initiativen sowohl im Innenverhältnis als auch mit anderen internationalen Maßnahmen koordiniert werden sollen. Diese Problematik schlägt sich vor allem im Bereich der momentan noch stark fragmentierten Finanzierungsinstrumente nieder. Die Agrarpolitik ist im Klima- und Energiepaket weitgehend unberücksichtigt geblieben, obwohl in diesem Politikfeld große Potentiale für die Reduktion von Treibhausgasemissionen vorhanden sind. Eine direkte Ausstrahlung in das internationale Umfeld, gar eine Führungsrolle ist hier nicht zu erkennen. 
Die EU hat es demnach bislang nur ansatzweise geschafft, anderen Staaten die Überlegenheit bestimmter Lösungsansätze für das Problem des Klimawandels zu demonstrieren. In allen untersuchten Politikfeldern kann die EU weitere Anstrengungen unternehmen, um die internationale Klimapolitik richtungsweisend voranzubringen.

\section{Bibliografia}

Ayers J., Huq S., Chandani A. (2010), Assesing EU Assistance for Adaption to Climate Change in Developing Countries: A Southern Perspective, in: The New Climate Policies of the European Union: International Legislation and Climate Diplomacy, Hrsg. S. Oberthür, M. Pallemaerts, C. Roche Kelly, Brüssel, S. 231-250.

Birger Skjaerseth J., Wettestad J. (2010), The EU Emissions Trading System Revised, in: The New Climate Policies of the European Union: International Legislation and Climate Diplomacy, Hrsg. S. Oberthür, M. Pallemaerts, C. Roche Kelly, Brüssel, S. 65-91.

Boulanger P., Messerlin P. (2009), Long-Term Challenges Facing European Agricul ture: The Need for Public and Private Policies, German Marshall Fund of the United States, Washington.

Bundesministerium für wirtschaftliche Zusammenarbeit und Entwicklung (2010), Die Aid-Effectiveness-Agenda - Wirksamkeit der Zusammenarbeit steigern. Zuletzt aufgerufen am 16. Juni 2010, http://www.bmz.de/de/ziele/ziele/parisagenda/index.html.

EurActiv (2010a), EU-Aktionsplan zu Energieeffizienz. Zuletzt aufgerufen am 20. Mai 2010, http://www.euractiv.com/de/energieeffizienz/eu-aktionsplan-energieeffizi enz/article-143289.

EurActiv (2010b), EU-Energieminister gegen strengere Effizienz-Ziele. Zuletzt aufgerufen am 5. Juni 2010, http://www.euractiv.de/energie-klima-und-umwelt/artikel/eu-energieminister-gegen-mehr-energieeffizienz-003177.

EurActiv (2010c), EU-Politik für Erneuerbare Energien. Zuletzt aufgerufen am 5. Mai 2010, http://www.euractiv.com/de/energie/eu-politik-erneuerbare-energien/article-145024.

Europäische Kommission (2006), Proposal for a Directive of the European Parliament and of the Council Establishing a Framework for the Protection of Soil and Amending Directive 2004/35/EC, Brüssel, COM(2006) 232 final.

Europäische Kommission (2008), Mitteilung zum Richtlinienpaket ,Erneuerbare Energiequellen und Klimawandel“, Büssel. Memo/08/33 vom 23. Januar.

Europäische Kommission (2009a), Commission Staff Working Document: The role of Eu ropean agriculture in climate change mitigation, Brussels, European Union.

Europäische Kommission (2009b), A Template for National Renewable Energy Action Plans under Directive 2009/28/EC of the European Parliament and of the Council, Brüssel.

Europäische Kommission (2009c), Verordnung (EG) Nr. 244/2009 der Kommission vom 18. März 2009 zur Durchführung der Richtlinie 2005/32/EG des Europäischen Parlaments und des Rates im Hinblick auf die Festlegung von Anforderungen an die umweltgerechte Gestaltung von Haushaltslampen mit ungebündeltem Licht, Amtsblatt der EU, L 76/3.

Europäische Kommission (2010), Erneuerbare Energien: Prognosen zeigen EU auf Weg 20\%-Ziel zu erreichen, Brüssel.

Europäischer Rat (2007), Schlussfolgerungen des Vorsitzes vom 8./9. März 2007, Brüssel.

Europäisches Parlament und Rat der EU (2003), Richtlinie 2003/97/EG des Europäischen Parlaments und des Rates vom 10. November 2003 zur Angleichung der Rechtsvor schriften der Mitgliedstaaten für die Typgenehmigung von Einrichtungen für indi rekte Sicht und von mit solchen 
Einrichtungen ausgestatteten Fahrzeugen sowie zur Änderung der Richtlinie 70/156/EWG und zur Aufhebung der Richtlinie 71/127/EWG, Amtsblatt der EU, L 25/1.

Europäisches Parlament und Rat der EU (2005), Richtlinie 2005/32/EG des Europäischen Parlaments und des Rates vom 6. Juli 2005 zur Schaffung eines Rahmens für die Festlegung von Anforderungen an die umweltgerechte Gestaltung energiebetrie bener Produkte und zur Änderung der Richtlinie 92/42/EWG des Rates sowie der Richtlinien 96/57/EG und 2000/55/EG des Europäischen Parlaments und des Rates, Amtsblatt der EU, L 191/29.

Europäisches Parlament und Rat der EU (2006), Richtlinie 2006/32/EG des Europäischen Parlaments und des Rates vom 5. April 2006 über Endenergieeffizienz und Energie dienstleistungen und zur Aufhebung der Richtlinie 93/76/EWG des Rates, Amtsblatt der EU, L 114/64.

Europäisches Parlament und Rat der EU (2009a), Entscheidung Nr. 406/2009/EG des eu ropäischen Parlaments und des Rates vom 23. April 2009 über die Anstrengungen der Mitgliedstaaten zur Reduktion ihrer Treibhausgasemissionen mit Blick auf die Erfüllung der Verpflichtungen der Gemeinschaft zur Reduktion der Treibhausgase missionen bis 2020, Amtsblatt der EU, L 140/136.

Europäisches Parlament und Rat der EU (2009b), Richtlinie 2009/28/EG des Europäischen Parlaments und des Rates vom 23. April 2009 zur Förderung der Nutzung von Energie aus erneuerbaren Quellen und zur Änderung und anschließenden Auf hebung der Richtlinien 2001/77/EG und 2003/30/EG, Amtsblatt der EU, L 140/16.

Europäisches Parlament und Rat der EU (2009c), Richtlinie 2009/29/EG des Europä ischen Parlaments und des Rats vom 23. April 2009 zur Änderung der Richtlinie 2003/87/EG zwecks Verbesserung und Ausweitung des Gemeinschaftssystems für den Handel mit Treibhausgasemissionszertifikaten, Amtsblatt der EU, L 140/63.

Fischer S (2009), Die Neugestaltung der EU-Klimapolitik: Systemreform mit Vorbildcharakter?, „Internationale Politik und Gesellschaft“ 2009, Nr. 2, S. 108-126.

Germanwatch (2008), Ein großer Schritt für die Politik, ein zu kleiner für das Klima. Zuletzt aufgerufen am 3. März 2010, http://www.germanwatch.org/presse/2008-01-23.htm.

Grubb M., Gupta J. (2000), Leadership. Theory and Methodology, in: Climate Change and European Leadership. A Sustainable Role for Europe?, Hrsg. J. Gupta, M. Grubb, Dordrecht, S. 15-24.

Gupta J., Persson A., Olsson L. (2010), Mainstreaming Climate Change in Develop ment Co-operation: Conditions For Success, in: Making Climate Change Work For Us: European Perspectives on Adaptation and Mitigation Strategies, Hrsg. M. Hulme, H. Neufeldt, Cambridge, S. 319-339.

Gupta J., Ringius L. (2001), Climate Leadership: Reconciling Ambition and Reality, „International Environmental Agreements“ 1(2): S. 281-299.

Haug C., Berkhout F. (2010), Abschied von Kyoto+? Die EU und das Trauma von Kopenhagen, „Welttrends“ 18(73).

Howes T. (2010), The EU'S New Renewable Energy Directive (2009/28/EC), in: The New Climate Policies of the European Union: Internal Legislation and Climate Diplomacy, S. Oberthür, M. Pallemaerts, Brüssel, S. 117-150.

Klima-Allianz (2010), EU Gipfel 17.-18. Juni: Energieeffizienz verbindlich machen und die EU-Treibhausgasreduktion auf $30 \%$ anheben. Berlin.

Lederer M. (2010) Klimapolitik zwischen Kyoto und Cancún, „Welttrends“ 18(73), S. 23-32.

Lindenthal A. (2009), Leadership im Klimaschutz. Die Rolle der Europäischen Union in der internationalen Umweltpolitik, Frankfurt.

Malnes R. (1995), 'Leader' and 'Entrepreneur'in International Negotiations: A Conceptual Analysis, „European Journal of International Relations“ 1(1), S. 87-112. 
Michaelowa A (1998), Impact of Interest Groups on EU Climate Policy, „Environmental Policy and Governance" 8(5), S. 152-160.

Michaelowa A. (2006), Kann die EU ihre Vorreiterrolle in der internationalen Klimapolitik glaubhaft fortsetzen?, in: Die Europäische Gemeinschaft in der internationalen Umweltpolitik, Hrsg. P-C. Müller-Graf, E. Pache, D. H. Scheuing, Baden-Baden, S. 169-183.

Núnez F. J., Egenhofer C., Behrens A. (2009), For a Future Sustainable, Competitive and Greener EU Budget. Integrating the Climate Change Objectives of the EU, Brüssel, Centre for European Policy Studies: Final Report of a CEPS Task Force.

Oberthür S., Pallemaerts M. (2010), The EU's Internal and External Climate Policies: an Historical Overview, in: The New Climate Policies of the European Union: Internal Legislation and Climate Diplomacy, Hrsg. S. Oberthür, M. Pallemaerts, Brüssel, S. 27-63.

Oberthür S., Roche Kelly C. (2008), EU Leadership in International Climate Policy: Achievements and Challenges, „The International Spectator“ 43(3), S. 35-50.

Olesen J. E., Bindi M. (2002), Consequences of Climate Change for European Agricultural Productivity, Land Use and Policy, „European Journal of Agronomy“ 16(4), S. 239-262.

Peskett L., Grist N., Hedger M., Lennartz-Walker T., Scholz I. (2009), Climate Change Challenges For EU Development Co-operation: Emerging Issues, European Development Co-operation to 2020 .

Rat der EU (2010), Council Conclusions: Towards a New Energy Strategy for Europe 2011-2020, Brüssel. 3017th Transport, Telecommunications and Energy Council Meeting.

Roche Kelly C., Oberthür S., Pallemaerts M. (2010), Introduction, in: The New Climate Policies of the European Union: Internal Legislation and Climate Diplomacy, Hrsg. S. Oberthür, M. Pallemaerts, Brüssel, S. 11-25.

Schreurs M. A., Tiberghien Y. (2007), Multi-Level Reinforcement: Explaining European Union Leadership in Climate Change Mitigation, „Global Environmental Politics“ 7(4), S. 19-46.

Schröder D. (2010), EU sagt Duschköpfen den Kampf an. Zuletzt aufgerufen am 22. Mai 2010, http://www.spiegel.de/wirtschaft/service/0,1518,674834,00.html.

Shrivastava M. K., Goel N. (2010), Shaping the Architecture of Future Climate Governance: Perspectives From the South, in: Global Climate Governance Beyond 2012: Architecture, Agency and Adaptation, Hrsg. F. Biermann, P. H. Pattberg, F. Zelli, Cambridge, S. 116-134.

Skodvin T., Andresen S. (2006), Part One: Negotiating International Environmental Regimes - Leadership Revisited, „Global Environmental Politics“ 6(3), S. 13-27.

Underdal A. (1994), Leadership Theory: Rediscovering the Arts of Management, in: International Multilateral Negotiation: Approaches to the Management of Complexity, Hrsg. I. W. Zartman, San Francisco, CA, S. 178-197.

United Kingdom Parliament (2008), Select Committee on European Scrutiny, 2nd Report of Session 2007-2008.

United Nations Framework Convention on Climate Change (2007), Report on the Analysis of Existing and Potential Investment and Financial Flows Relevant to the Development of an Effective and Appropriate International Response to Cliamte Change, Dialogue Working Paper. Wien.

Vogler J., Stephan H. R. (2007), The European Union in Global Environmental Governance: Leadership in the Making, „International Environmental Agreements“ 7(4), S. 389-413.

Wissenschaftlicher Beirat der Bundesregierung Globale Umweltveränderungen (2010), Klimapolitik nach Kopenhagen: Auf drei Ebenen zum Erfolg, Politikpapier, Berlin, S. 6.

Young O. R. (1989), The Politics of International Regime Formation-Managing Natural-Resources and the Environment, „International Organization“ 43(3), S. 349-375. 
Young O. R. (1991), Political-Leadership and Regime Formation - on the Development of Institutions in International Society, „International Organization“ 45(3), S. 281-308.

\section{Streszczenie}

\section{Ukierunkowana rola przywódeza Unii Europejskiej w międzynarodowej polityce klimatycznej}

Od szczytu klimatycznego w Kopenhadze w grudniu 2009 r. Unia Europejska próbowała przejąć inicjatywę w zakresie formułowania celów i zadań dla ochrony światowego klimatu i walki z zanieczyszczeniami powietrza. Ze względu na opór wielu państw nakreślone ambitne plany redukcji gazów cieplarnianych nie zostały powszechnie zaakceptowane, ale sama UE może już poszczycić się pewnymi osiagnięciami. Olbrzymią słabością w koncepcjach UE jest Wspólna Polityka Rolna, gdzie postęp w walce z zanieczyszczeniami ziemi i powietrza jest najmniejszy. Dla wielu państw członkowskich wdrażanie unijnych rozporządzeń wiąże się z olbrzymimi kosztami, których te kraje nie są w stanie udźwignąć. Istnieją nadal różnego rodzaju przeszkody administracyjne, które również utrudniają wprowadzenie jednolitych norm ochrony klimatu. 